\title{
One more second
}

\section{Every now and then, an extra second is added to an earthly year - a cause for trouble and debate, as Felicitas Arias has been witnessing.}

$\mathrm{D}$ uring the last 16 years, part of my work has been invested in the neverending leap-second discussion. In spite of the strong and divergent positions of different groups, there has been an evolution in the debate, and there is good hope for finding a solution in about seven years' time. But what is all the fuss about?

The leap second was born as a witty solution for synchronizing the unchanging rhythm of atomic time, measured with unprecedented accuracy, with the time derived from the irregular (and generally decreasing) rotation rate of the Earth. This happened in the late 1960s, as a consequence of the adoption of the new definition of the second as "the duration of 9,192,631,770 periods of the radiation corresponding to the transition between the two hyperfine levels of the ground state of the ${ }^{133} \mathrm{Cs}$ atom"1.

Before, the provision of practical timescales and the definition of the second had been the task of astronomers, with decisions made by the International Astronomical Union (IAU) and supported by other relevant organizations. Universal Time was the general name for the time linked to the rotation of the Earth, representing mean solar time, as recommended by the IAU in $1948^{2}$. This timescale was meant to have the same reading all over the world, and far from being just a tool for astronomers, it became the timescale used for international time coordination.

In 1968, the General Conference on Weights and Measures voted for the adoption of the definition of the second involving hyperfine levels of ${ }^{133} \mathrm{Cs}$. Two years later, International Atomic Time (TAI) was officially born.

The choice for a practical timescale was a matter of discussion at the International Radio Consultative Committee, presently the International Telecommunication Union (ITU) Radiocommunication Sector. Its task was to establish and operate a worldwide service of standard frequencies and time signals. These signals were principally used for maritime navigation, for which, just as for astronomers, the Earth's rotation time is essential. Various atomic timescales existed around 1955. In particular, the Bureau International de l'Heure put in place a procedure including time steps and frequency corrections to relate atomic time to the Earth's rotational time. It was considered disadvantageous to adopt two different timescales, one for maritime navigation, astronomy and civil applications (derived from the Earth's rotation), and another for metrology (uniform atomic time). As a result, Coordinated Universal Time (UTC) came to life - defined as identical in rate to TAI, except for the insertion of one second whenever necessary to compensate for the effect of the slowing down of the Earth's rotational velocity. To guarantee that UTC provides a good approximation to UT1 (Universal Time corrected for polar motion), a tolerance of not more than $0.9 \mathrm{~s}$ was fixed to the difference between UT1 and UTC. A recommendation adopted by the ITU in 1970 on standard frequencies and time signal emissions fully describes the procedure of leap-second insertions ${ }^{3}$.

Since the length of a mean solar day is about 2.5 milliseconds longer than a day of 86,400 SI seconds, we can expect a difference of about one second in a year. The Earth's rotation is gradually slowing down, with additional random fluctuations. Since 1999 the frequency of leap-second insertions has decreased, implying that in the future it will become necessary to insert leap seconds more frequently.

The main problem with the leap second is that it is not compatible with time synchronization with a precision better than one second, and the development of global satellite navigation systems threatened the reputation of UTC. These systems avoid leap-second insertions and maintain uniform and continuous internal atomic timescales for their operation, which are often used as time references instead of UTC. Computers are used for the dissemination of time, but they have difficulties with accepting a second numbered ' 60 ', putting at risk the use of computers as time-service devices.

The leap-second saga has certainly been the most controversial discussion in the 150 years of existence of the ITU. Previous changes of the practical timescale were based on technical discussions where the most relevant international organizations were involved. The debate that has taken place at the ITU since 2000 is mostly based on political, societal and cultural issues, together with claims of retro-compatibility of systems that sooner or later will be obsolete anyway. Proposals of maintaining two timescales disseminated in parallel have been supported by some administrations with the intent of preserving the leap second, but without any consideration for the consequences this could have on time coordination.

The issue was on the agenda of the 2015 ITU World Radiocommunication Conference, but it only concluded with a clarification of missions and responsibilities and a call to international organizations to collaborate on new studies ${ }^{4}-$ a missed opportunity to dismiss the leap second once and for all. UTC will still feature leap seconds until the 2023 conference. And so, depending on whether you are ahead or behind UTC, your next New Year's Eve or Day will last one second longer.

FELICITAS ARIAS is at the International Bureau of Weights and Measures, Pavillon de Breteuil, 92310 Sèvres, France.

e-mail: farias@bipm.org

\section{References}

1. Terrien, J. Metrologia 4, 41-45 (1968).

2. IAU VIIth General Assembly - Transactions of the IAU Vol. VII B: Proceedings of the 7th General Assembly (ed. Oort, J. H.) (Cambridge Univ. Press, 1950).

3. Recommendation ITU-R TF.460-6 (ITU, 2002); http://go.nature. $\mathrm{com} / 2 \mathrm{eXbVNR}$

4. Final Acts WRC-15, World Radiocommunication Conference (ITU, 2015); http://go.nature.com/2fm7mL8

$\mathrm{m} e \AA \mathrm{s} \mathrm{u} R E_{\mathrm{h}}$
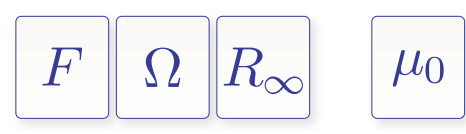

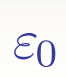

$\alpha$

$\sigma$

